\title{
Una nueva época para la Revista Internacional de Sistemas
}

\section{A new era for the Revista Internacional de Sistemas}

\author{
Sanz, M.T \\ Departamento de Didáctica de la Matemática \\ Universidad de Valencia \\ Valencia, Spain \\ m.teresa.sanz@uv.es
}

La Revista Internacional de Sistemas es una revista transdisciplinar que trata de dar cabida a todos aquellos autores que tengan la Dinámica de Sistemas como metodología en sus investigaciones.

Fue Jay W. Forrester (1961) quién desarrolló la Dinámica de Sistemas en el Massachussets Institute of Technology (MIT) como metodología transdisciplinar con la que construir modelos dinámicos de sistemas complejos y usarlos como herramienta de intervención en los mismos.

Uno de los objetivos de este marco metodológico es proporcionar a las ciencias sociales y humanísticas un estatus epistemológico similar al de las ciencias positivas (física, química, biología, economía, ...). Un camino para contribuir a potenciar cambios en estas ciencias es llegar a validar modelos dinámicos matemáticos con variables de interés social de la misma forma que se realiza en las ciencias positivas.

Es por ello que esta revista da la bienvenida a trabajos de interés mutuo para científicos dedicados tanto a las ciencias sociales como a las ciencias positivas, que pueden fomentar conexiones fructíferas entre ambas disciplinas.

La Revista Internacional de Sistemas se publica desde el año 1979 cuando Rafael Rodríguez Delgado la funda. Por ella han pasado diferentes directores como Rafael Rafael Beneito (1994-1999) o Antonio Caselles (1999-2016). Este último mi padre en la investigación decide poner en mis manos la gran responsabilidad de dirigir esta revista y es desde Enero de 2017 que tomo el cargo de Directora y Editora.

Hasta el momento la revista se publicaba en papel y los números más recientes se alojaban en la página web personal de Rafael Pla, sistémico profesor titular de la Universidad de Valencia. Pero era el momento de cambiar. Entramos en una nueva era para esta revista, tratando de abrir sus puertas y exponiéndola al mundo. Para ello se tramitó el alojamiento en el servidor para revistas digitales que nos proporciona la Universidad de Valencia (ojs.uv.es). y se adapta su formato para una indexación.

Este es el primer número que lanzo, y no puedo más que agradecer a todas aquellas personas que lo han hecho posible, desde los técnicos de la Universidad de Valencia, los informáticos amigos argentinos, y como no los autores que se prestaron a realizar esos primeros artículos que tratan de dar luz de lo que esta revista es. 